\title{
Dissipation of turbulence by magnetohydrodynamic shock waves
}

\author{
Andrew Lehmann and Mark Wardle \\ Department of Physics and Astronomy, and Research Centre in Astronomy, Astrophysics and \\ Astrophotonics Macquarie University \\ 2109, NSW, Sydney \\ email: andrew.lehmann@mq.edu.au
}

\begin{abstract}
We characterise steady, one-dimensional fast and slow magnetohydrodynamic (MHD) shocks using a two-fluid model. Fast MHD shocks are magnetically driven, forcing ions to stream through the neutral gas ahead of the shock front. This magnetic precursor heats the gas sufficiently to create a large, warm transition zone where all fluid variables only weakly change in the shock front. In contrast, slow MHD shocks are driven by gas pressure where neutral species collide with ion species in a thin hot slab that closely resembles an ordinary gas dynamic shock.

We computed observational diagnostics for fast and slow shocks at velocities $v_{s}=2-4 \mathrm{~km} / \mathrm{s}$ and preshock Hydrogen nuclei densities $n_{\mathrm{H}}=10^{2-4} \mathrm{~cm}^{-3}$. We followed the abundances of molecules relevant for a simple oxygen chemistry and include cooling by $\mathrm{CO}, \mathrm{H} 2$ and $\mathrm{H}_{2} \mathrm{O}$. Estimates of intensities of ${ }^{12} \mathrm{CO}$ rotational lines show that high- $\mathrm{J}$ lines, above $\mathrm{J}=6 \rightarrow 5$, are more strongly excited in slow MHD shocks.
\end{abstract}

Keywords. (magnetohydrodynamics:) MHD, shock waves, ISM: clouds, ISM: molecules

\section{Introduction}

The character of star formation is intimately related to the supersonic magnetohydrodynamic (MHD) turbulent dynamics of the giant molecular clouds in which stars form (Mac Low \& Klessen 2004). A significant amount of turbulent energy dissipates in low velocity shock waves (Stone et al.1998, Smith et al.2000a, Smith et al.2000b). In MHD fluids the three families of shocks - fast, intermediate and slow-differ in how they compress and heat the molecular gas, and so their radiative signatures probe distinct physical conditions (Kennel et al.1989). No study has identified which of these families controls the turbulent dissipation in clouds, and so we compare the structural and observational differences between fast and slow shocks in order to motivate such a classification.

\section{Shock structure}

In Figure 1 we show example temperature and cooling profiles of typical fast and slow shocks in molecular clouds. Both shocks propagate at $3 \mathrm{~km} / \mathrm{s}$ into gas with preshock Hydrogen nuclei density $n_{\mathrm{H}}=10^{3} \mathrm{~cm}^{-3}$. The preshock magnetic field is $10 \mu \mathrm{G}$ for the fast shock and $80 \mu \mathrm{G}$ for the slow shock. The fast shock is magnetically driven, with a high magnetic pressure postshock region forcing ion species through the neutral gas. Thus the heating timescale in fast shocks is determined by the slow ion-neutral collision timescale. The slow shock is gas pressure driven, with a high gas pressure postshock region forcing neutral species through the ions. Thus the heating timescale in slow shocks is determined by the fast neutral-ion collision timescale. The cooling timescale of the gas lies between these two scales and so slow shocks are able to reach higher peak temperatures, as can be seen by the temperature profiles in Figure 1. The high peak temperature $(\sim 500 \mathrm{~K})$ in the slow shock drives chemical reactions and a strong $\mathrm{H}_{2}$ cooling rate which is negligible in the cooler $(\sim 50 \mathrm{~K})$ fast shock. 

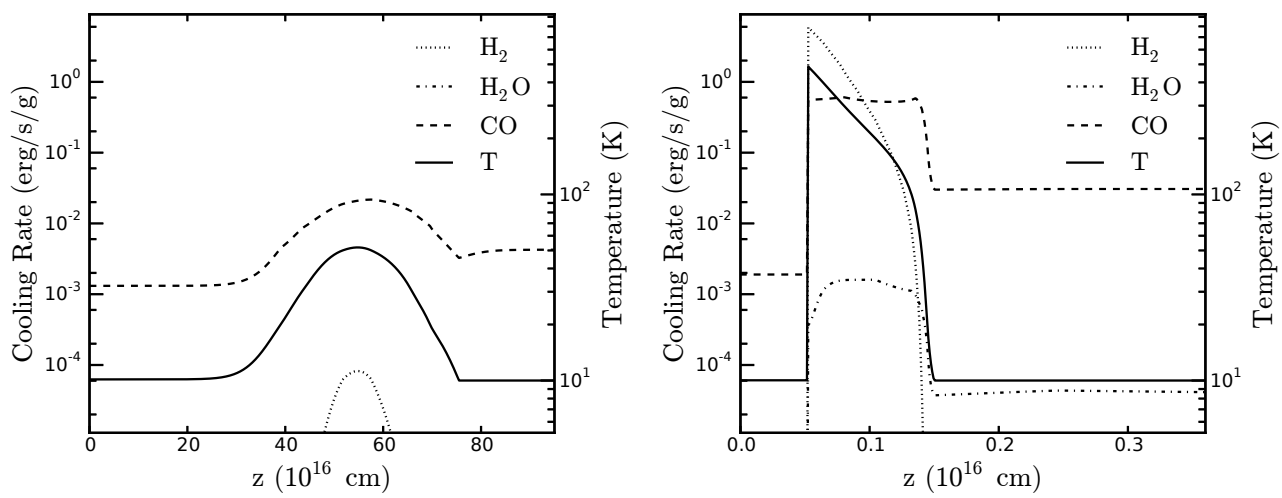

Figure 1. Temperature and cooling profiles of a fast (left) and slow (right) MHD shock. Both shocks travel at $3 \mathrm{~km} / \mathrm{s}$ and propagate into gas with preshock Hydrogen nuclei density $n_{\mathrm{H}}=10^{3} \mathrm{~cm}^{-3}$.

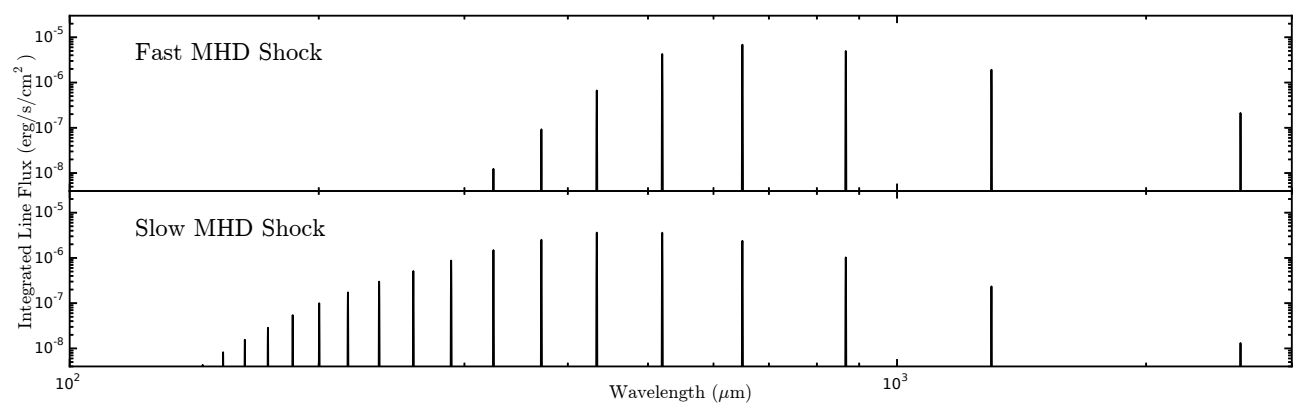

Figure 2. RADEX estimates of integrated line fluxes from rotational lines of ${ }^{12} \mathrm{CO}$ for the fast (top) and slow (bottom) shocks shown in Figure 1.

\section{Observational differences}

We use RADEX, a non local thermodynamic equilibrium radiative transfer code to estimate integrated line intensities from ${ }^{12} \mathrm{CO}$ (van der Tak et al.2007). Figure 2 shows these lines for the fast and slow MHD shocks plotted in Figure 1. This shows that the high temperatures reached in slow shocks excite high- $J{ }^{12} \mathrm{CO}$-above $J=6 \rightarrow 5$-more strongly than in fast shocks. This result holds throughout the velocity range $v_{s}=2-4 \mathrm{~km} / \mathrm{s}$ and preshock density range $n_{\mathrm{H}}=10^{2-4} \mathrm{~cm}^{-3}$, making these lines strong indicators of shock type.

\section{References}

Kennel C. F., Blandford R. D., Coppi P. 1989, J. Plas. Phys., 42, 299

Mac Low M.-M., Klessen R. S. 2004, Reviews of Modern Physics, 76, 125

Smith M. D., Mac Low M.-M., Heitsch F. 2000a, A\&A, 362, 333

Smith M. D., Mac Low M.-M., Zuev J. M., 2000b, A\&SA, 356, 287

Stone J., Ostriker E., Gammie C. 1998, ApJ, 508, L99

van der Tak F. F. S., Black J. H., Schöier F. L., Jansen D. J., van Dishoeck E. F. 2007, $A \& A$, 468,627 